\title{
Epistemological challenges in contemporary Scandinavian healthcare systems exemplified by people's widespread use of complementary and alternative medicine
}

\author{
Anita Salamonsen MSc, $\mathbf{P h D}^{1}$ and Rolf Ahlzén, $\mathbf{M D}, \mathbf{P h D}^{2}$ \\ 1e-mail: anita.salamonsen@uit.no \\ The National Research Center in Complementary and Alternative Medicine, Department of \\ Community Medicine, Faculty of Health Sciences, UiT The Arctic University of Norway, N- \\ 9037 Tromsø \\ 2e-mail: Rolf.Ahlzen@kau.se, phone +46702519517 or +4654611446 \\ Psychiatric Open Care Unit, Karlstad hospital, 65185 Karlstad, Sweden \\ Directory Unit, University Hospital of Örebro, 70185 Örebro, Sweden
}

Published in Health: An Interdisciplinary Journal for the Social Study of Health, Illness and Medicine, 2017, doi: 10.1177/1363459317693408

Biographical note:

Anita Salamonsen is a senior researcher at the National Research Center in Complementary and Alternative Medicine, Department of Community Medicine at UiT The Arctic University of Norway. Salamonsen holds a Phd in medical sociology and is a lecturer in relational perspectives on healthcare, doctor-patient communication and qualitative/mixed research methods. Her research mainly examines patients' and medical doctors’ perspectives, including multi-disciplinary studies of quality and trust in healthcare systems, decisionmaking, patient-provider communication, and risk perception.

Rolf Ahlzén is a GP and psychiatrist, working part time in psychiatric open care. He also has a background in history and philosophy and is associate professor of medical humanities at the University of Karlstad in Sweden. He has been involved with medical ethics consulting and the teaching of medical humanities for over thirty years. 


\begin{abstract}
Modern Western public healthcare systems offer predominantly publicly subsidized healthcare traditionally based on biomedicine as the most important basis to cure persons who suffer from disorders of somatic or psychiatric nature. To which extent this epistemological position is suitable for this purpose is under scientific debate and challenged by some people's personal understandings of health and illness, their individual illness experiences and their decision-making. Current studies show decreasing levels of patient trust in Western public healthcare and a widespread patient-initiated use of complementary and alternative medicine (CAM) which often is linked to unmet patient-defined healthcare needs. Patients'/CAM users' understandings of their afflictions are often based on elements of biomedical knowledge as well as embodied and experience-based knowledge. We believe this points to the need for a phenomenologically and socially based understanding of health and illness. In this article, we analyze challenges in contemporary healthcare systems, exemplified by people's widespread use of CAM and based on three ways of understanding and relating to unhealth: disease (the biomedical perspective), illness (the phenomenological perspective) and sickness (the social perspective). In public healthcare systems aiming at involving patients in treatment processes, acknowledging the co-existence of differing epistemologies may be of great importance to define and reach goals of treatment and compliance.
\end{abstract}

\title{
Keywords
}

Complementary and alternative medicine; Experiencing illness and narratives, Health policy; Patient-physician relationship; Phenomenological approaches 


\section{Introduction}

In modern Western welfare states such as Scandinavian countries, UK and Canada, citizens who become ill are supposed to trust and receive healthcare within healthcare systems that are mainly publicly subsidized. Such public healthcare systems may be perceived as fragmented and highly specialized biomedical systems (Clarke 2014; Fadlon, 2004; Kuhnle, 1999; Shuval and Mizrachi 2004; ZZ et al., 2015), with a strong focus on the cause of a disease, the characteristics of the organ involved and the current treatment for a recognized pathology (Cunningham and Wilson, 2011). The relevance of such a biomedical perspective as the main epistemological position for the treatment of disease is currently being challenged by person-based perspectives of health and illness. This concept has several connotations, but we here assume that it means an understanding of the nature of disease that brings biological factors, as well a subjective experience and sociocultural context into consideration. The latter perspectives are typically found in social science evaluations of public healthcare systems, public health policy documents, and - last, but not least - in patients who increasingly express their personal healthcare needs, healthcare experiences and high expectations for healthcare services (Bowling et al., 2012).

No one paradigm of medicine or system of healthcare any longer holds an uncontested monopoly in modern societies, however dominant biomedicine may seem (Adams et al., 2012). The experience-based, unregulated, widespread and patient-initiated use of complementary and alternative medicine (CAM) may, among other things, be linked to possible epistemological weaknesses in the biomedical perspective, as may decreasing levels of trust in modern public health systems (Holmboe et al. 2011; YY and Author 1, 2013). Exploring and discussing such basic epistemological matters, underlying healthcare practices, may be central in achieving a higher degree of healthcare goal attainment - that is, 
relieving suffering by promoting health, meeting the ethical demand to sometimes cure, often alleviate and always console.

\section{Objectives}

This article provides an overview and discussion of the differing and often competing epistemological assumptions that can be recognized in contemporary Western landscapes of healthcare. We analyze challenges in contemporary healthcare systems, exemplified by people’s widespread and most often experience-based use of CAM and based on three ways of understanding and relating to unhealth: disease (the biomedical perspective), illness (the phenomenological perspective) and sickness (the social perspective). In public healthcare systems aiming at involving patients in treatment processes, acknowledging the co-existence of differing epistemologies may be of great importance to define and reach goals of treatment and compliance. Tacit “taken for granted” attitudes deeply affect personal and professional understandings of health and illness, and thus the content and quality of education and clinical practice as well as patients’ decision-making.

\section{CAM use in public healthcare contexts}

What constitutes CAM varies with a number of factors, and thus the terms "CAM" and "CAM user” have been defined differently between studies (Kristoffersen et al., 2008). Furthermore, what is defined as CAM changes over time as the borders between biomedically based public healthcare and CAM are constantly changing. The Cochrane Collaboration has defined CAM as

“... a broad domain of healing resources that encompasses all health systems, modalities and practices, and their accompanying theories and beliefs, other than those intrinsic to the politically dominant health system” (Zollman and Vickers, 1999, p. 693). 
Norway is one of very few countries where CAM is legally defined. The Norwegian Act No. 64 of 27 June 2003 relating to the alternative treatment of disease, illness, and so forth (Lovdata, 2004) defines CAM as:

... health related treatment which is practiced outside the established health services and which is not practiced by authorized health personnel. However, treatment practiced within the scope of the established health services or by authorized health personnel is also covered by the term alternative treatment when the methods used are essentially methods that are used outside the established health services.

No applicable official definitions of CAM exist in the rest of Scandinavia, UK or Canada, but the situation is quite similar to Norway. Recent studies show that as many as $50 \%$ of Scandinavians have used CAM occasionally or regularly (Fønnebø, 2013; Johannessen and Ostenfeld-Rosenthal, 2012). A systematic review of studies of CAM use in 15 Western countries including Canada, the US, Sweden and Denmark, found that the use was substantial and stable in all the included countries, e.g., about $50 \%$ Australia and about 37 \% in the US (Harris et al., 2012). The prevalence of CAM use seems to be higher among middle-aged women with higher education, and people with poor self-reported health (Bishop and Lewith, 2010; Hanssen et al., 2005). CAM users do not seem to leave the public healthcare system despite negative experiences that sometimes function as push-factors for their decision to use CAM (e.g., Author 1 et al. 2010, 2012a, 2012b; Author 1 2013; Danell 2015; Ekholm and Kjøller, 2007; Launsø, 1996; Skovgaard et al., 2014, Verhoef et al., 2006). The concept of CAM includes different forms of use of CAM and commitment to CAM, a fact that should be acknowledged when analyzing the meaning and importance of CAM use (Kristoffersen et al., 2008). A consumer commitment model (ZZ et al., 2016) and 
a model that distinguishes between CAM users visiting a CAM practitioner repeatedly and those who are not that dedicated (Kristoffersen et al., 2008) have thus been suggested. Another possibly important distinction has been made between the majority of seriously ill CAM users who choose to use CAM as supplement to recommended treatment offered in the public healthcare system and a smaller group choosing to use CAM as an exclusive alternative despite medical advice (Author 1 et al., 2012; Author 1, 2013). The concept of CAM thus refers to a diverse field of practices, products, knowledge, and technologies recognized in late modern societies (Coulter, 2004). “CAM use” may vary from taking commonly used herbs and vitamins as supplements to treatments offered in the public healthcare system, to the decision to forgo important biomedical cancer treatment (Author 1 et al., 2012; Author 1, 2013). However, as argued by Adams and colleagues (2012), Siahpush (1998) and other scholars, many mind-body oriented CAM modalities such as spiritual healing that are highly valued by their users, still operate largely outside the mainstay of public healthcare systems and have traditionally been excluded from the core medical curriculum.

The widespread use of CAM has been interpreted both as a result of positive experiences with CAM , and a somewhat negative attitude toward the biomedical dominance in Western public healthcare systems (e.g., Author 1 et al., 2010, 2012a, 2012b; Author 1, 2013; Coulter, 2004; Coulter and Willis 2007; Launsø, 1996; Mulkins et al., 2015; Rakel et al., 2012; Sarsina et al., 2012; Siahpush 1998). Siahpush (1998) distinguishes in an interesting review between three competing hypotheses to explain why people turn to CAM despite receiving or being offered treatment within the public healthcare system: 
- The medical outcome hypothesis postulates that people turn to CAM because they are dissatisfied with the health outcomes of treatments offered in the public healthcare system

- The medical encounter hypothesis states that people are dissatisfied with the interaction with doctors and the public healthcare system

- The postmodern hypothesis explains peoples’ commitment to CAM by the recent emergence of a new value system that is congruent with the philosophical underpinnings of CAM (Siahpush, 1998).

These hypotheses clearly overlap and are supported by a large number of empirical studies. Such patterns of CAM use are often found in persons living with chronic conditions who seek relaxation and well-being when full cure is not possible (e.g., Author 1 et al., 2010; Author 1 et al., 2012; Author 1, 2013, 2015b; Eisenberg et al., 1993; Skovgaard et al., 2012). Central elements in many CAM modalities have been associated with person-centeredness, empowerment, salutogenic care and self-management (e.g., Author 1, 2015b; Berger et al., 2012; Launsø, 1996; Maciocia, 1994; Mulkins et al., 2015; Rakel et al., 2012).

Complementary explanations for CAM use can be found in the literature, such as increasing economic capital (Eisenberg et al., 1993), and CAM users perceived as consumers (e.g., Lupton, 1997; ZZ et al. 2016). We would like to add that it seems likely that increasing expectations among Western populations for well-being and quality of life create an increasing demand for therapies in addition to what the predominant healthcare systems are able to offer.

According to the World Health Organization, the general goal of a healthcare system is "to promote, restore or maintain health” (WHO 2007, p. 2). Such broadly defined ideals are challenged by the fact that there are significant variations in the interpretations of the 
concept of health across individuals, professions, organizations and cultures (ZZ et al., 2015). The widespread use of CAM may be perceived as an important demonstration of a large group of healthcare users' health and illness understandings and their healthcare preferences. Systematic reviews have shown that beliefs about control and participation, a holistic view of health, and a desire for natural, non-invasive treatments, predict CAM use after controlling for demographic and clinical variables. Studies have also revealed that many CAM users experience their health beliefs as conflicting compared to those of health professionals in the public healthcare (Author et al., 2010; Author 1, 2013, 2015b; Berger et al., 2012; Bodeker et al., 2007; Hughner and Kleine, 2008; Launsø,1996; Mao et al., 2008; Mulkins et al., 2015; Rakel et al., 2012; Verhoef et al., 2006). Recent white papers like the Norwegian innovation program Future Care (Report to the Storting, 2012-2013) recommend a higher degree of patient involvement and shared decision-making in public healthcare. It may remain challenging, however, to change the everyday clinical practice in line with these ideals. The conditions for and consequences of such an idea of increased self-determination and involvement must also be carefully scrutinized. We propose that knowledge gained from the experiences of active and involved CAM users and analyses of CAM use in Western welfare states may provide us with useful information.

\section{Three main perspectives on health and illness}

We assume that a biomedical, a social, and a phenomenological perspective coexist in different contaminations and with varying emphasis among persons seeking healthcare as well as among healthcare professionals and CAM practitioners. These perspectives mirror a fundamental epistemological divide in present society, a tension that has been with Western societies since the $17^{\text {th }}$ century, when Descartes attempted to formulate his answer to the 
mechanistic challenge of the new science. The mind-body dualism pervades contemporary society, as well as a tension between individual and society, subject and social group. It is important to emphasize that the three perspectives we discuss are theoretical constructions and analytical tools that are more rigid than what can be found in real life. Doctors of course know that they always relate to persons in their clinical practice, and they are trained in doctor/patient-relationship. Patients/CAM users of course may have both positive and negative experiences with both healthcare professionals and CAM practitioners who are not medically trained, and who are not always necessarily so person oriented. The biomedical perspective presented here is a theoretical template, an ideal type in the Weberian sense, for modern medicine, which can in practice never be followed. Still, as epistemological model the presented perspective exerts strong influence. Many students experience that they are encouraged to "choose" one or the other perspective to become a “real” medical doctor or medical sociologist (Cunningham and Wilson, 2011; Williams, 2006).

\section{The biomedical perspective: Focus on "the real disease"}

Western public healthcare systems are since early $19^{\text {th }}$ century to a gradually higher extent relying on a biomedical basis for medical practice, research and education. Biomedicine here refers to "the commonly established form of Western medicine taught in medical schools that occupies a dominant, often exclusive monopoly over legitimate medical care in many societies” (Shuval and Mizrachi, 2004, p. 676). The biomedical perspective arose in conjunction of scientific approaches to understanding the human body, where disease was defined as tissue dysfunction and structural deviances in organ systems. Without doubt, it has significantly influenced major medical advances in diagnosis and treatment. Biomedicine is focused on the cause of a disease, the characteristics of the organ involved, and the current 
evidence based treatment for a recognized pathology. Disease is here understood as "negative bodily occurrences as conceived of by the medical profession” (Hofmann, 2002, p. 657). Critique has since at least forty years been raised against the dominance of this perspective, stressing the risk that subjective experiences of illness as well as socioeconomic determinants of health may be brushed aside in clinical practice. A typical critique focuses on the displacement of the ill person's experiences in the professional understanding of diseases as it is:

(...) discovered by research, that diseases exist independently of persons, and that the correct attitude or 'stance' of the doctor is that of objective scientist”. (...) These uncontested assumptions have led to a black-or-white dichotomy between correct and incorrect medical practice, without consideration of each patient's ideas, beliefs or particular context, and how these factors can impact on disease outcomes.

(Cunningham and Wilson, 2011, p. 450)

An important element in this "impersonal" system is a perspective on the body and the self as separate entities. Diseases are located in the body, and are (at least in principle) measurable and depictable and have identifiable causes. This is within medical sociology and theory of science referred to as medicine's mind/body dualism and the doctrine of specific aetiology (Nettleton, 2006). Disease is considered independently from context, individual experiences, and subjective interpretations (Hoenders et al., 2008; Shuval and Mizrachi, 2004). Within this scope, social relationships (typically physician-patient) are formalized, and impersonal. The body is described within a biologically based framework and often regarded as a complex biological machine, Health and recovery is perceived as dependent on the body's physical existence, and personality is accorded little emphasis when considering diagnosis and treatment (Annandale, 1998; Bury, 2005; Hofmann, 2002; Manning and Fabrega 1973; Nettleton, 2006). 
Admittedly, this perspective is stereotyped, for the obvious reason that in its pure form, it could not work in clinical practice. However, as we earlier noted, as a basic model and ideal of scientific rigor, it exerts considerable influence and thus creates more or less strong tensions in practice with the two other perspectives, to which we now turn.

\section{The phenomenological perspective: Focus on the individual illness experience}

The phenomenological perspective focuses on the individual illness experience, i.e., "illness is negative bodily experiences as conceived of by the person himself” (Hofmann 2002, p. 657). 
The concept of illness here involves action, choice, and interpretation. This is often described as "the patient perspective", or "first person perspective” on health and illness. This implies that the patients are perceived as acting subjects, reacting to their illness through interpretations formed by earlier experiences and those they interact with (e.g., physicians). Epistemologically, understanding the patient's illness is considered to rely on the patient's own experience-based knowledge. We here understand experience to mean the total sum of a person's perceptions of something being wrong in the body and his/her cognitive reflections and emotional response to this (Author 2, 2008).

In phenomenology, “the lived body” is essential. According to Turner (1995), phenomenology attempts an exploration of the relationship between the objectiveinstrumental body ("the body-as-nature”) and the subjective-living body ("the lived body”). Turner (1995) refers to the philosopher Merleau-Ponty and outlines very interestingly how a body-in- the-life-world phenomenology perceives the body as our access to the world, which in this approach in return creates and affects the body in different ways. Such coexistence involves an open, communicative situation in which the boundaries between self and others perceived as interwoven. In relation to the biological body, Leder (1998, p. 125) describes how this phenomenological approach

... does not so much replace the biological account, as place it within a broader perspective ... Anatomy and physiology of the lived body are always intertwined with the body's intentionality in ways that undermine facile claims of priority. Just as our physical structure lays the groundwork for our mode of being-in-the-world, so our interactions with this world fold back to reshape our body in ways conducive to health and illness. A medicine of the lived body dwells in this intertwining. 


\section{Sickness: Focus on the social context}

However, there seems to be a close link between illness as the unique subjective experience, and sickness, as the socially mediated understanding and acting that results from, and also shapes, the illness experience. A social context is so to speak, incorporated by the experiencing subject. Such an understanding of the concept of experience is inspired by theorists like Alfred Schutz and especially John Dewey as: “(...) they both take the social and intersubjective world of human agency as the point from which a notion of experience should be developed” (Grøn, 2004, p. 109).

Illness is, in the social-constructivist perspective, negotiated in the social space, typically in the doctor-patient relationship, but also in family and among friends and at the working place. Many social constructivists in the field of medical sociology have expressed a strong critique of biomedicine and the biomedical perspective along the lines mentioned above. In turn, the constructivist perspective has been criticized for implying that "the world, and the body, and disease within it, becomes equated or conflated with our discursive constructions” (Williams, 2006, p. 9). Furthermore, critics point out that there could be a discursive determinism in relation to the meaning of the biological body if everything is reduced to the social as power/knowledge (Williams, 2006). Sickness may thus be defined as “negative bodily occurrences as conceived of by the society and/or its institutions” (Hofmann, 2002, p. 657). We are inclined to see illness and sickness as two sides of the same coin, mutually dependent and closely interacting. For heuristic purposes, they may be held apart.

\section{Disease and illness/sickness: Differences in treatment contexts and healthcare needs}


However much it is argued that the mind-body dichotomy should be replaced by a more unified conception of human beings, it remains remarkably insistent to such attempts. It can be recognized in notions like psychosomatic, and plays tricks with clinical encounters when no organic deviances are discovered but the patient has an over-whelming illness experience. Because of this still dualistic foundation of clinical practice, it is essential that those who attempt to heal persons experiencing unhealth are aware of these epistemologically contrary understandings of the nature of disease/illness. We argue that it is of crucial importance in future education and clinical practice to emphasize that the task is to deal with biological processes (diseases) as well as mental phenomena (interpretations, experiences, i.e. illness) in their sociocultural context (sickness).

It must be underscored that the classical divide between the biomedical focus on disease and cure and the phenomenological/social focus on the life with illness does not necessarily represent a problem. Both experiencing and treating acute illness are clearly different from experiencing and treating chronic states. From the patient perspective, problems occur when such differences in time frames and contexts are not acknowledged. The attention of patients living with chronic illnesses is often turned from treatment and cure to management and care (Bury, 2001). From a sociological perspective, this development illustrates how both medical doctors in clinical encounters, as well as public healthcare systems, have to approach many patients' lay perspectives on health, illness, and treatment (Bury, 2001), as this interest is obviously of strong relevance for both diagnosis, treatment and care - and for the organization of healthcare systems. 
Some scholars within the CAM field strongly argue that such epistemological gaps as those we have described as the biomedical, phenomenological and social perspectives on health and illness do not exist (Hoenders et al., 2008). Instead of different perspectives, they focus on healthcare as complex, pluralistic, and constantly changing. This may be mainly a question of how concepts are used. Fadlon (2004, p. 69) posits that the domestication of CAM over the last decades has been followed by "a process of cultural negotiation in which both the dominant narrative of biomedicine as well as that of CAM changed”, and that CAM "has become fashionable as part of the postmodern spectacle of the body and has developed into a new means of medical surveillance” (ibid.). Various understandings of CAM’s epistemological position exist, strongly influenced by factors like culture, context, and the characteristics of the various CAM modalities. As we have noted, CAM is an extremely heterogeneous phenomenon, which makes it hard to generalize about. However, we insist that there still are considerable epistemological differences between CAM and the biomedically based public healthcare systems in Western countries. CAM is in general associated with a more holistic, in the sense of taking many factors into account, approach to illness, which again can be linked to a phenomenological and social understanding of health and illness (Bishop et al., 2007; Coulter, 2004; Danell, 2015; Hoenders et al., 2008; Launsø,1996; Rakel et al., 2012; Shuval and Mizrachi, 2004).

\section{What can phenomenological and social perspectives on health and illness add to medical education and clinical practice?}

Healthcare users and healthcare systems are constantly changing, often in directions not foreseen by public health authorities. The encounter between lay experiences and medical knowledge can for analytical purposes be described as a potential clash between biomedical 
and phenomenological/social understandings of health and illness, if not healthcare professionals are able to integrate several epistemological perspectives in their clinical practice. We consider Western patients' widespread use of CAM to be an interesting example of the postmodern trend toward a plurality of subjective (lay) discourses and illness behaviors, although science is "the most culturally embedded and ubiquitous system of knowledge in contemporary society” (McClean and Shaw, 2005, p. 745). The increasing use of CAM during the last decades may, to some extent, be perceived as a patient critique of public healthcare systems and the biomedical perspective on health and illness. It may also be perceived as an orientation toward a more individualized and experience-based understanding It has been argued that the widespread interest in and use of CAM indirectly contributes to the foundation of person-centered medicine, where the unique person is positioned at the center of healthcare and the therapeutic process (e.g., Author 1, 2015b; Bodeker et al., 2007; Danell 2015; Sarsina et al., 2012; Mao et al., 2008; Verhoef et al., 2006).

In a phenomenological perspective, symptoms of disease are always interpreted through the lens of a person’s individual life story, his/her memories, hopes, fears, beliefs and desires. These are of course also partly socially formed. From this perspective, illness reaches deep into our lives, raising questions and interpretations that may move beyond scientific explanations (Antonovsky, 1979, 1987). Cassell was one of the first to point to the inherent weakness of the predominating clinical epistemology:

Suffering is experienced by persons, not merely by bodies, and has its source in challenges that threaten the intactness of the person as a complex social and psychological entity. Suffering can include physical pain but is by no means limited to it. The relief of suffering and the cure of disease must be seen as twin obligations of a medical profession that is truly dedicated to the care of the sick. (Cassell, 1982, p. 639) 
Another example of such an approach to healthcare is the salutogenic thinking (Antonovsky, 1979, 1987), which focuses on man’s complete history, including disease and coping resources - what can contribute to better health and well-being. When people face a diagnosis, they will, in Antonovskys’ perspective,, also seek an understanding of the existential meaning of the disease. Furthermore, Antonovsky $(1979,1987)$ claims that a person with a "sense of coherence" will have a high degree of autonomy and be oriented toward new knowledge. He/she will take what happens to his/her body and health as a challenge and try to master it. Antonovsky also points to the fact that medical knowledge is constantly changing, and introduces a hypothesis that a sense of coherence may have physiological consequences. He thus asks whether research in psychoneuroimmunology may assist in understanding the biological processes through which the sense of coherence influences health (Antonovsky, 1987). The widespread and continuing use of CAM in many Western countries has been explained in terms of self-care and holistic approaches to health and illness (e.g., Author 1 et al. 2010, 2012a, 2012b; Baarts and Pedersen, 2009; Coulter and Willis, 2007; Danell, 2015; Eisenberg, 1993; Siahpush, 1998, ZZ et al., 2016; Verhoef et al., 2006). Furthermore, CAM use has been interpreted as a counter-trend against "biomedicalization” (Clarke, 2014) and a contribution to personalization and prevention of illness (Author 1 2015b; Danell 2015; Sarsina et al., 2012). Antonovsky claims that in order for patients in public healthcare to experience control and that their own efforts can make a difference, the patients' active approach has to be socially valued. He challenges public healthcare systems and requests doctors to ask themselves whether they promote a positive or negative development of the sense of coherence (Antonovsky, 1979, 1987). Rakel and colleagues (2012) link a salutogenic focus to CAM education and argue for several potential benefits of salutogenic education also in public healthcare, such as more efficient healthcare with reduced cost and healthier and happier healthcare professionals. 
On the contrary, Antonovsky argues that the possibility that there may be knowledge and treatment approaches outside the public healthcare system can be an impetus for further action and give a feeling of control and that personal efforts can affect the outcome of the disease (Antonovsky 1979, 1987). Other sociologists have argued that alternative medicine disempowers the individual and that the growth of CAM represents a paradigmatic shift from biomedicine that is not positive. CAM may for instance be perceived as an aspect of the postmodern medicalization-trend, where people seek help for any signs of un-health (Coulter and Willis, 2007; Siahpush, 1999). Antonovsky (1987) also points to a possible risk of introducing the salutogenic model of health into public healthcare because making everything in a person's life medically relevant can increase medicalization and social control of people.

The need for complementary epistemologies in future public healthcareWe argue that the patient perspective should be better integrated into public healthcare and medical education. Both biomedical, phenomenological and social knowledge is crucial to meet a patient's healthcare needs. The epistemologies that form the basis of the biomedical and the hermeneutic/phenomenological perspective should thus be combined, and even integrated into public healthcare and education in the future. Such an endeavor has the potential to contribute substantially to the reduction and prevention of suffering due to disease, and the three perspectives should thus be perceived as complementary rather than contradictory approaches in clinical encounters and education. Such a coexistence opens a possibility to make patients more content with their encounters when ill, making diagnosis more accurate and treatment more efficient. Such potentials yet to carry out may also represent important resources to strengthen the quality of education for healthcare professionals who must handle the complex meeting between so-called person-centered care and highly specialized biomedical treatment.

It has been argued that personalized care still seems out of reach in modern Western public healthcare systems, and that one important reason for this is poorly understood tensions 
between personalized and professional goals of care that are inherent to professionalism and specialization (Author 1 et al. 2016; ZZ et al., 2015). The professional duty in shared decision-making processes will thus be to translate patients' personal goals into goals that are realistic professional goals (ZZ et al., 2015). It is also of crucial importance to approach the question whether this may inadvertently lead us to an unjustifiably broad understanding of the tasks of medicine (Author 2, 2011), and to discuss and develop ethically based constraints to avoid further medicalization. If the ideals heavily emphasized in recent Western white papers are to be taken seriously, we need to actively reflect over existing epistemologies in education and clinical practice. Phenomenological and social perspectives may add important knowledge to strengthen person-centered healthcare, while biomedical perspectives may remind us that human beings belong to both nature and culture and that biology may restrict social actions and aspirations, such as patient involvement and shared decision-making. Integration of CAM in hospitals within public healthcare systems is increasing in practice, although not much discussed on a system level (Jacobsen et al., 2015). Evaluations of integration initiatives have discussed paradigmatic clashes as well as practical challenges (e.g., Coulter, 2004). Situations where there is a clash between personal goals and professional goals of care, such as when cancer patients decide to use alternative treatments only (Author 1 et al., 2012, 2016; Author 1, 2013, 2015a; Coulter 2004), will remain an ethical and practical dilemma to health professionals (Starr and Zawacki, 1999; ZZ et al., 2015).

A relevant question is "how lay” the personally collected knowledge on which many users of public healthcare and CAM base their treatment decisions actually is. A mental health study revealed that: “Lay theories, perceptions and explanations ... are constructed and negotiated in an increasingly plural and complex environment of knowledge” (Kangas 2001, p. 89). Patients may themselves become the "experts" in heterogeneous, context-specific dimensions 
of knowledge (Author 1 2015a, 2015b; Pedersen and Baarts, 2010). Some of them - but indeed not all - may be described as explorers of healthcare irrespective of healthcare systems, and with reflexive and pragmatic illness behaviors influenced by culture and context (Lupton, 1997; Author 1 et al., 2010; Author 1 2015b; Pedersen and Baarts 2010). Still, the probably strongest cultural force in relation to illness and disease is so-called school medicine. Prior (2003) points out that lay people are experts by virtue of having experience, but that "experience on its own is rarely sufficient to understand the technical complexities of disease causation, its consequences and its management” (p. 53). Medical concept theories pervade present society, a process that has been called medicalization, and which seems to be going on at an increasing speed (Conrad, 2005).

A further development of a person-centered, yet largely biomedically based medicine would raise multiple scientific, ethical and practical issues. First and foremost; what should be considered valid knowledge and information in different healthcare and education settings, when perspectives collide? Moreover, to which extent and how should these perspectives actually be implemented into the training of healthcare professionals? What about situations where there is a clash between personal and professional goals of care? What do medical ethics actually imply with regard to medical responsibility if patient involvement in decisionmaking and treatment is considered a leading principle? To find answers to such crucial questions is of course demanding and challenging. Still, the fact that such questions currently are under debate, is indeed a promise for the future of person-centered, yet largely biomedically based public healthcare systems.

\section{Conclusion}


The highly specialized biomedical knowledge incorporated in current modern Western public healthcare systems is of crucial importance to patients' health and well-being. These systems are officially aiming at patient involvement and shared decision-making. There is still, however, a rather strong focus on biomedical knowledge as the dominant basis for problem solving. Patients report unmet healthcare needs, and their widespread use of CAM may be seen as a sign of shortcomings of the exclusive dominance of the biomedical perspective. We thus propose that a crucial question for the future is whether complementary epistemological perspectives will be considered valuable in science and clinical practice. The growth of interest in CAM is a reminder to public healthcare systems and healthcare professionals that there are individual needs that seem to pass unmet and that seem to fall outside the present dominant frames of understanding. If experience-based knowledge and patients' self-defined healthcare needs are disregarded in public health policy and medical education, this may negatively influence modern healthcare users' trust in and interaction with public healthcare systems. Consequently, the use of unregulated, expensive and underexplored alternatives found in CAM may increase. This may in turn have multiple possible negative effects on patient safety and the legitimacy and quality of public healthcare, as trust and risk is closely connected. Furthermore, we claim that the need to widen the epistemological frames of sciences and clinical practice may indeed be seen as an invitation to bridge the gap between "the two cultures" - that of the sciences and that of the humanities. Clinical practice will prevent, cure, ameliorate and console by using a broad scope of human knowledge and experience. In so doing, the healthcare needs of the $21^{\text {st }}$ century will stand better chances to be met. 


\section{References}

Adams J et al. (2012) Introduction. In Adams J. et al. (eds) Traditional, complementary and integrative medicine. An international reader. Hampshire: Palgrave Macmillan, pp. 15.

Annandale E (1998) The sociology of health and medicine. A critical introduction.

Cambridge: Polity Press.

Antonovsky A (1979) Health, stress, and coping. San Francisco: Jossey-Bass.

Antonovsky A (1987) Unraveling the mystery of health: How people manage stress and stay well. San Francisco: Jossey-Bass.

Author 1 et al. (2010).

Author 1 et al. (2012a).

Author 1 et al. (2012b).

Author 1 (2013).

Author 1 (2015a).

Author 1 (2015b).

Author 1 et al. (2016)

Author 2 (2008).

Author 2 (2011).

Baarts C and Pedersen IK (2009) Derivative benefits: Exploring the body through complementary and alternative medicine. Sociology of Health \& Illness 31: 719-733.

Berger S et al. (2012) The health professional-patient-relationship in conventional versus complementary and alternative medicine. A qualitative study comparing the perceived use of shared decision-making between two different approaches of medicine. Patient Education and Counseling 88: 129-137. 
Bergh A. and Bjørnskov C (2011) Historical trust levels predict the current size of the welfare state. KYKLOS 64(1): 1-19.

Bishop FL et al. (2007) A systematic review of beliefs involved in the use of complementary and alternative medicine. Journal of Health Psychology 12(6):851-67.

Bishop FL and Lewith GT (2010) Who uses CAM? A narrative review of demographic characteristics and health factors associated with CAM use. Evidence-Based Complementary and Alternative Medicine 7(1):11-28.

Bodeker G et al. (2007) Policy and public health perspectives in traditional, complementary and alternative medicine: An overview. In G. Bodeker and Burford G (eds) Traditional, complementary and alternative medicine. Policy and public health perspectives. London: Imperial College Press, pp. 9-40.

Bowling A et al. (2012) The measurement of patients' expectations for health care: A review and psychometric testing of a measure of patients' expectations. Health Technology Assessment 16(30) i-xii doi 10.3310/hta16300

Broom A (2009) Intuition, subjectivity, and Le Bricoleur: Cancer patients' accounts of negotiating a plurality of therapeutic options. Qualitative Health Research 19(8): 1050-1059.

Broom A and Adams J (2012) A critical social science of evidence-based healthcare. In A. Broom \& J. Adams (Eds.). Evidence-based healthcare in context. England: Ashgate, pp.1-19.

Bury M (2001) Illness narratives: Fact or fiction. Sociology of Health and Illness 23(3): 263285.

Bury M (2005) Health and Illness. Cambridge: Polity Press. 
Cassell EJ (1982) The nature of suffering and the goals of medicine. New England Journal of Medicine 306(11): 639-645.

Clarke AE (2014) Biomedicalization. The Wiley Blackwell Encyclopedia of Health, Illness, Behavior, and Society, 137-142.

Conrad P (2005). The Shifting Engines of Medicalization. Journal of Health and Social Behavior; 46 (March): 3-14.

Coulter I (2004) Integration and paradigm clash: The practical difficulties of integrative medicine. In Tovey P et al. (eds) The mainstreaming of complementary and alternative medicine. London: Routledge, pp. 103-122.

Coulter I and Willis E (2007) Explaining the growth of complementary and alternative medicine. Health Sociology Review, 16(3-4): 214-225.

Cunningham W and Wilson H (2011) Complaints, shame and defensive medicine. Postgraduate Medical Journal, 87(1034): 837-840.

Danell JA (2015) From disappointment to holistic ideals: a qualitative study on motives and experiences of using complementary and alternative medicine in Sweden. Journal of Public Health Research, 4: 538

Davis EM (2008) Risky Business: Medical discourse, breast cancer, and narrative. Qualitative Health Research 18(65): 65-76.

Eisenberg DM et al. (1993) Unconventional medicine in the United States: Prevalence, costs, and patterns of use. New England Journal of Medicine 328: 246-252.

Ekholm O and Kjøller M (2007) The use of alternative treatment in Denmark: Results of the nationally representative Health and Morbidity study 2005. [In Danish: Brugen af alternativ behandling i Danmark: resultater fra den nationalt repræsentative Sundhedsog sygelighedsundersøgelse 2005.] Tidsskrift for Forskning i Sygdom og Samfund 6: $15-24$. 
Fadlon J (2004) Meridians, chakras, and psycho-neuro-immunology: The dematerializing body and the domestication of alternative medicine. Body \& Society 10(4): 69-86. Fønnebø V (2013) Rapport: Alternativ behandling i Norge 2012. [In English: Report: Alternative treatment in Norway 2012.] National Research Center in Complementary and Alternative Medicine, UiT The Arctic University of Norway.

Gerhardt U (1989) Ideas about illness: An intellectual and political history of medical sociology. New York: University Press.

Grøn L (2004) Winds of change, bodies of persistence. Health promotion and lifestyle change in institutional and everyday contexts. Ph. D. thesis, Aarhus, Denmark: University of Aarhus.

Hanssen B et al. (2005) Use of complementary and alternative medicine in the Scandinavian countries. Scandinavian Journal of Scandinavian Health Care 23: 57-62.

Harris PE et al. (2012) Prevalence of complementary and alternative medicine (CAM) use by the general population: a systematic review and update. International Journal of Clinical Practice 66 (10); 924-939.

Hoenders $\mathrm{H}$ et al. (2008) Western and alternative medicine: A comparison of paradigms and methods. Journal of Alternative and Complementary Medicine 14: 894-896.

Hoffman B (2002) On the triad disease, illness and sickness. Journal of Medicine and Philosophy 27(6): 651-673.

Holmboe O et al. (2011) The 2011 Commonwealth Fund survey among sicker adults: Results from a comparative survey in 11 countries. [In Norwegian: Commonwealth Fundundersøkelsen i 2011 blant utvalgte pasientgrupper: Resultater fra en komparativ undersøkelse i 11 land.] Rapport 18/2011. Oslo: Nasjonalt kunnskapssenter for helsetjenesten. 
Hughner RS and Kleine SS (2008) Variations in lay health theories: Implications for consumer health care decision making. Qualitative Health Research, 18(12): 16871703.

Jacobsen R et al. (2015) Use of complementary and alternative medicine within Norwegian hospitals. BMC Complementary and Alternative Medicine, 15(1). doi:

10.1186/s12906-015-0782-5.

Kangas I (2001) Making sense of depression: Perceptions of melancholia in lay narratives. Health 5(1): 76-92.

Kristoffersen AE. Fønnebø V and Norheim AJ (2008). Use of complementary and alternative medicine among patients: Classification criteria determine level of use. The Journal of Alternative and Complementary Medicine 14(8), 911-919.

Kuhnle S (1999) The Scandinavian welfare state in the global competitive society. [In Norwegian: Den skandinaviske velferdsstat i det globale konkurransesamfunn.] In Does the welfare state work? [In Norwegian: Virker velferdsstaten?] Stortingsmelding nr. 50 98/99 - Utjamningsmeldinga.

Launsø L (1996) The alternative treatment field. Use and development; rationality and paradigms. [In Danish: Det alternative behandlingsområde. Brug og udvikling; rasjonalitet og paradigmer.] København: Akademisk Forlag.

Leder D (1998) A tale of two bodies: The Cartesian corpse and the lived body. In Welton, D. (ed) Body and flesh: A philosophical reader. Oxford: Blackwell, pp. 35-50.

Lovdata: Act No. 64 of 27 June 2003 relating to alternative treatment of disease, illness, etc. www.regjeringen.no/globalassets/upload/kilde/hod/red/2005/0076/ddd/pdfv/242593regulations_no_1501-forskr_alt.behandl_.pdf(accessed 29.01.2016) 
Lupton D (1997) Consumerism, reflexivity and the medical encounter. Social Science \& Medicine 45(3): 373-381.

Manning K and Fabrega H (1973) The experience of self and body: Health and illness in the Chiapas Highlands. In G. Psathas (ed) Phenomenological Sociology. London: Wiley \& Sons.

Mao JJ et al. (2008) Cancer survivors with unmet needs were more likely to use complementary and alternative medicine. Journal of Cancer Survivorship 2: 116-124.

McClean S and Shaw A (2005) From schism to continuum? The problematic relationship between expert and lay knowledge. An exploratory conceptual synthesis of two qualitative studies. Qualitative Health Research 15(6): 729-749.

Maciocia G (1994) The practice of Chinese medicine. Edinburgh: Churcill Livingstone.

Mulkins A et al. (2015) From the conventional to the alternative: Exploring patients' pathways of cancer treatment and care. Journal of Complementary and Integrative Medicine doi: 10.1515/jcim-2014-0070.

Nettleton S. (2006) The sociology of health and illness. Cambridge: Polity Press.

Pedersen IK and Baarts C (2010) “Fantastic hands” - but no evidence: The construction of expertise by users of CAM. Social Science \& Medicine 71: 1068-1075.

Prior L (2003) Belief, knowledge and expertise: The emergence of the lay expert in medical sociology. Sociology of Health \& Illness 25: 41-57.

Rakel D et al. (2012) Complementary and alternative medicine education: Promoting a salutogenic focus in health care. In Adams J et al. (eds) Traditional, complementary and integrative medicine. An international reader. Hampshire: Palgrave Macmillan, pp. 176-182.

Report to the Storting 29 (2012-2013) Morgendagens omsorg [Future Care]. Oslo: The Norwegian Ministry of Health and Care Services; 2013. 
Rowe R. and Calnan M. (2006). Trust relations in health care - the new agenda. European Journal of Public Health, 16(1): 4-6.

Sarsina R et al. (2012) Traditional, complementary and alternative medical systems and their contribution to personalisation, prediction and prevention in medicine-personcentred medicine. The EPMA Journal 3(15) doi:10.1186/1878-5085-3-15.

Shuval JT and Mizrachi N (2004) Changing boundaries: Modes of coexistence of alternative and biomedicine. Qualitative Health Research, 14(5): 675-690.

Siahpush M. (1999) A sociological critique of alternative medicine: Liberation or disempowerment? Social Alternatives, 18(4): 57-62.

Siahpush M (1998) Postmodern values, dissatisfaction with conventional medicine and popularity of alternative therapies. Journal of Sociology 34(1): 58-70.

Skovgaard LE et al. (2014) Exclusive use of alternative medicine as a positive choice: a qualitative study of treatment assumptions among people with multiple sclerosis in Denmark. International Journal of MS Care 16(3): 124-131.

Skovgaard LE et al. (2012) Use of complementary and alternative medicine among people with multiple sclerosis in the Nordic countries. Autoimmune Diseases doi:10.1155/2012/841085

Starr J and Zawacki BE (1999) Voices from the silent world of doctor and patient. Cambridge Quaterly of Healthcare Ethics 8(2): 129-138.

Stub T et al. (2015) Mapping the risk perception and communication gap between different professionals of healthcare providers in cancer care: a cross-sectional protocol. $B M J$ Open doi: 10.1136/bmjopen-2015-008236

Stratton TD and McGivern-Snofsky JL (2008) Toward a sociological understanding of complementary and alternative medicine use. The Journal of Alternative and Complementary Medicine, 14(8): 777-783. 
Turner B (1995) Medical power and social knowledge. London: Sage Publications.

Verhoef M et al. (2006) Evaluating complementary and alternative medicine interventions: in search of appropriate patient-centered outcome measures. BMC Complementary and Alternative Medicine 6(38) doi:10.1186/1472-6882-6-38

Williams SJ (2006) Medical sociology and the biological body: Where are we now and where do we go from here? Health 10(1): 5-30.

YY and Author 1 (2013).

ZZ et al. (including Author 1) (2015).

ZZ et al. (including Author 1) (2016).

Zollman C and Vickers A (1999) ABC of complementary medicine: What is complementary medicine? British Medical Journal, 319: 693-696.

World Health Organization (2007) Everybody's business: Strengthening health systems to improve health outcomes: WHO’s framework for action. ISBN 9789241596077. 